\title{
A PSO Based Solution Methodology for Short Term Hybrid Generation Scheduling Problem
}

\author{
Shubham Tiwari ${ }^{1}$, Bharti Dwivedi ${ }^{2}$, M.P Dave ${ }^{3}$ \\ Research Scholar ${ }^{1}$, Professor ${ }^{2}$, Institute of Engineering and Technology, Lucknow, U.P., India. \\ Professor $^{3}$, Shiv Nadar University, Dadri, U.P., India
}

\begin{abstract}
A multiple stage solution methodology involving Priority List method and Particle Swarm Optimization Technique with Time Varying Acceleration Coefficients has been proposed for solving short term generation scheduling problem having ten thermal generating units and one solar generating unit. The per day revenue saving in operational cost after inclusion of $300 \mathrm{MW}$ solar plant is quantified. The proposed method is found reliable after executing the simulations several times.
\end{abstract}

Keywords:Unit Commitment Problem (UCP), Priority List Method (PLM), Particle Swarm Optimization with Time Varying Acceleration Coefficients (PSO_TVAC), Solar Energy Resources (SER), Economic Load Dispatch (ELD)

\section{INTRODUCTION}

Unit Commitment Problem (UCP) is an onerous non-linear optimization problem where load for a scheduled time frame is satisfied by generation with minimum cost undervarious constraints [1]. The rising demand of energy warrants inclusion of Renewable Energy Resources (RES) as fossil fuel resources are becoming scarce. Today energy saving associated with environmental protection has become prudent. The inclusion of RES into mainstream power system is inexorable because it can not only reduce the overall operating cost but can also prove to be sustainable and eco-friendly in nature [2-3]. In wake of this, efforts have been done to include solar generation with conventional thermal generation [4-6].

Particle Swarm Optimization (PSO) technique is found to be one of the most effective techniques in obtaining solution for rigorous optimization problems like UCP and Economic Load Dispatch (ELD) [7-9].The advantages of PSO are that it is robust, its parameter tuning is easy and it can be hybridized with other techniques available in literature [10-12]. The adaptability of PSO allows hybridizing Priority List Method (PLM) which is an effective classical solution technique for providing better feasible solutions to the problem [13-15].

\section{PROBLEM FORMULATION}

There are two sub sections where the modeling of thermal generation [14], [16] and solar generation [5] have been carried out.

(a) Thermal Generation:

The cost functionCost ${ }_{\mathrm{N}}$ is given by Equation (1).

$\operatorname{Cos} t_{N}=\sum_{i=1}^{N}\left[F C_{i}\left(P_{i h}\right)+S T C_{i}\left(1-U_{i(h-1)}\right)\right] U_{i h}$

$F C_{i}\left(P_{i h}\right)$ is the fuel cost of $\mathrm{i}^{\text {th }}$ unit with power output $\left(P_{i h}\right)$ at the $\mathrm{h}^{\text {th }}$ hour. FC is fuel cost function which is quadratic polynomial with coefficients $\mathrm{a}_{\mathrm{i}}, \mathrm{b}_{\mathrm{i}}$ and $\mathrm{c}_{\mathrm{i}}$.It is represented by Equation (2).

$F C_{i}\left(P_{i h}\right)=a_{i}+b_{i} P_{i h}+c_{i} P^{2} i h$

If the downtime of ${ }^{\text {ith }}$ unit is less than or equal to the summation of minimum down time $\left(\mathrm{MD}_{\mathrm{i}}\right)$ and the Cold start up time $\left(\mathrm{C}_{\mathrm{s}}\right)$, then the start-up cost of that unit $\left(\mathrm{STC}_{\mathrm{i}}\right)$ is taken as Hot start-up cost $\left(\mathrm{H}_{\mathrm{sc}}\right)$. Else it is taken as Cold start -up cost $\left(\mathrm{C}_{\mathrm{sc}}\right)$ representedby Equation (3).

$S T C_{i}=H s c_{i}$

When, $X_{i}^{\text {off }} \leq M D_{i}+C s$

$X_{i}{ }^{\text {off }}$ is the duration in which the $\mathrm{i}_{\text {th }}$ unit is continuously off. If the downtime of related unit is greater than the summation of minimum down time hours $\left(\mathrm{MD}_{\mathrm{i}}\right)$ and cold start-up hours $(\mathrm{Cs})$ then the cold start-up cost $\left(\mathrm{C}_{\mathrm{sc}}\right)$ is considered. 
This can be represented by Equation (4).

$S T C_{i}=\operatorname{Csc}_{i}$

When, $X_{i}^{\text {off }}>M D_{i}+C s$

Thus, the total cost $\left(\operatorname{Cost}_{\mathrm{NH}}\right)$ for the complete schedule of the given time frame is given by Equation (5).

$\operatorname{Cos} t_{N H}=\sum_{h=1}^{H} \sum_{i=1}^{N}\left[F C_{i}\left(P_{i h}\right)+S T C_{i}\left(1-U_{i(h-1)}\right)\right] U_{i h}$

$\mathrm{U}_{\text {ih }}$ is the ON/OFF status of the $\mathrm{i}^{\text {th }}$ unit at $\mathrm{h}^{\text {th }}$ hour. Digit ' 1 ' represents ON, while ' 0 ' represents the OFF status.

The constraints of UCP (only thermal) considered here are as follows.

1) Power Balance Constraint

$$
\sum_{i=1}^{N} P_{i h} U_{i h}=L D_{h}
$$

$P_{\text {ih }}$ is the generation in MW of $i^{\text {th }}$ unit in $h^{\text {th }}$ hour, $U_{\text {ih }}$ is the ON/OFF status of $i^{\text {th }}$ unit and $L D_{h}$ is the load demand at $\mathrm{h}^{\text {th }}$ hour.

2) Spinning Reserve Constraint

$$
\sum_{i=1}^{N} P_{i(\max )} U_{i h} \geq L D_{h}+S R_{h}
$$

$\mathrm{Pi}_{(\max }$ is the maximum generation in $\mathrm{MW}$ of $\mathrm{i}^{\text {th }}$ unit and $\mathrm{SR}_{\mathrm{h}}$ is the spinning reserve at $\mathrm{h}^{\text {th }}$ hour. In this paper for ten thermal generating units the spinning reserve is taken as $5 \%$ of total load.

3) Generation Limit Constraint

$$
P_{i(\min )} \leq P_{i h} \leq P_{i(\max )}
$$

4) Minimum up time constraints

$$
X_{i}^{\text {on }}(t) \geq M U_{i}
$$

5) Minimum down time constraint

$$
X_{i}^{\text {off }}(t) \geq M D_{i}
$$

6) Initial Status

It is the initial down time status that is required to be considered in the first hour of scheduling. The data regarding thermal generating units and load profile is given in Appendix I and II respectively.

(b) Solar Generation System

The power balance constraint under the lights of solar generation is given as

$$
\sum_{i=1}^{N}\left[P_{i}(t)+\right] U_{i h}+P_{\text {solar }}(t)=L D_{h}
$$

Where, $P_{\text {solar }}(t)$ is the hourly solar power output.The total availability of hourly solar power is calculated by using following equations [17].

$$
\begin{aligned}
& P_{\text {solar }}(s(t))=P_{\text {Sn }} \frac{s(t)}{s_{\text {stand. }}{ }^{*} R_{S}} ; 0 \leq s(t) \leq R_{S} \\
& P_{\text {solar }}(s(t))=P_{\text {sn }} \frac{s(t)^{2}}{s_{\text {stand. }}} ; s(t) \geq R_{S}
\end{aligned}
$$

Where, $\mathrm{s}(\mathrm{t})$ is forecasted solar radiation at hour $\mathrm{t}, \mathrm{S}_{\text {stand }}$ is solar radiation in standard environment taken as 1000 $\mathrm{W} / \mathrm{m}^{2}$ and $\mathrm{R}_{\mathrm{s}}$ is the cut-in radiation point set as $150 \mathrm{~W} / \mathrm{m}^{2}$ [17]. $\mathrm{P}_{\mathrm{sn}}$ is maximum generation capability of solar system taken as $300 \mathrm{MW}$. The data regarding radiation is provided in Appendix III. 


\section{SOLUTION METHODOLOGY}

A two stage solution methodology involving Priority Listing Method (PLM) and Particle Swarm Optimization with Time Varying Acceleration Coefficients (PSO_TVAC) is proposed for the solution of problem. The ON/OFF schedule in the first stage is determined by PLM and economic allocation of load among thermal generating units is done by PSO_TVAC.

\section{(a) Stage One-}

The priority vector is obtained by using equation $(26)[14,16]$.

$$
\text { priorityvector }=\frac{P_{(\max ), v e c}}{\max \cdot\left[P_{(\max ), v e c}\right]}+\frac{M D_{v e c}}{\max \cdot\left[M D_{\text {vec }}\right]}
$$

This priority vector is updated with the help of a pseudo code $[14,16]$ given below.

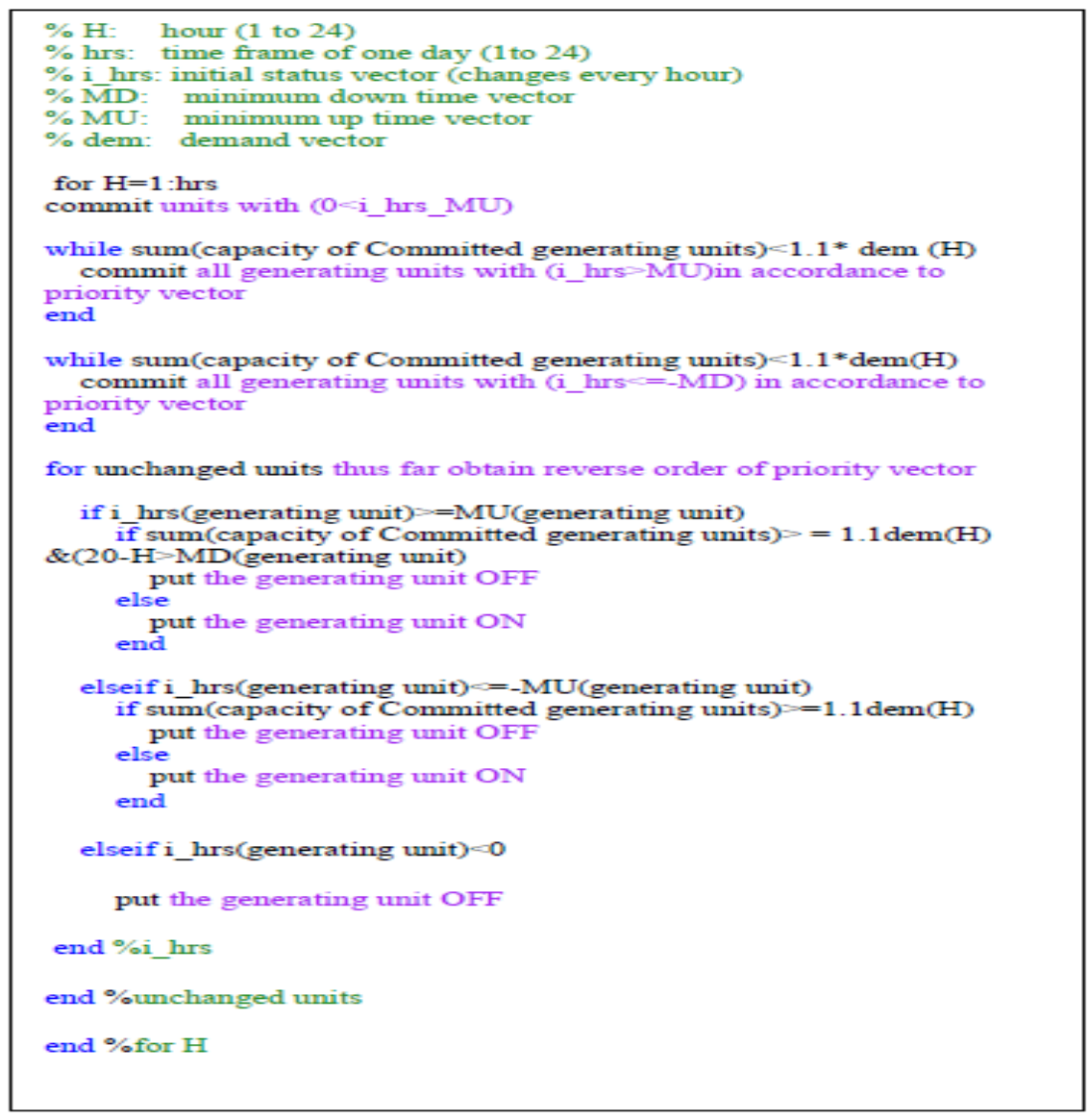

Fig.1. Pseudo code for obtaining ON/OFF Schedule

(b) Stage two-

The ON/OFF status obtained from stage one serves as an input to stage two where the load is distributed among thermal generating units economically. PSO_TVAC $[17,18]$ is proposed to solve non-linear UCP. The equations are as follows

$$
\begin{aligned}
& v_{i d}{ }^{(k+1)}=\left[W^{*} v_{i d}{ }^{k}+c_{1} * \operatorname{Rand}_{1}() *\left(P_{\text {bestid }}-x_{i d}{ }^{k}\right)+c_{2} * \operatorname{Rand}_{2}() *\left(G_{\text {best gd }}-x_{i d}{ }^{k}\right)\right] \\
& x_{\text {id }}{ }^{(k+1)}=x_{i d}{ }^{k}+v_{i d}{ }^{k+1}
\end{aligned}
$$

Where, ' $W$ ' is the inertia weight parameter which controls the global and local exploration capabilities of the particle. The linearly varying ' $W$ ' is given as

$$
w=w_{\max }-\left(\frac{w_{\max }-w_{\min }}{\text { iter }_{\max }}\right) * \text { iter }
$$


' $\mathrm{C}_{1}$ ' and ' $\mathrm{C}_{2}$ ' are acceleration coefficients, to deal with the high non-linear nature of UCP some modifications are done in classical PSO algorithm, in this paper PSO with Time Varying Acceleration Coefficients (TVAC) is used, the values of ' $\mathrm{C}_{1}$ ' and ' $\mathrm{C}_{2}$ ' can be given as

$$
\begin{aligned}
& c_{1}=\left(c_{1 f}-c_{1 i}\right) * \frac{\text { iter. }}{\text { iter }_{\text {max }}}{ }^{*} c_{1 i} \\
& c_{2}=\left(c_{2 f}-c_{2 i}\right) * \frac{\text { iter. }}{\text { iter } r_{\text {max }}} * c_{2 i}
\end{aligned}
$$

Where, $\mathrm{c}_{1 \mathrm{i}}, \mathrm{c}_{1 \mathrm{f}}, \mathrm{c}_{2 \mathrm{i}}, \mathrm{c}_{2 \mathrm{f}}$ are initial and final values of cognitive and social acceleration factors respectively. The values considered for acceleration coefficients are-

$$
c_{1 f}=0.5, c_{1 i}=2.5, c_{2 f}=2.5, c_{2 i}=0.5 \text {. }
$$

The bounds for velocity are set to make sure that the solution does not fly away. These bounds are set as explained below.

If, $\mathrm{V}_{\mathrm{id}}{ }^{(\mathrm{k}+1)}>\mathrm{V}_{\mathrm{d}}{ }^{\max }$, then, $\mathrm{V}_{\mathrm{id}}{ }^{(\mathrm{k}+1)}=\mathrm{V}_{\mathrm{d}}{ }^{\max }$

$$
\text { If, } V_{\text {id }}{ }^{(k+1)}<V_{d}{ }^{\min } \text {, then, } V_{\text {id }}{ }^{(k+1)}=V_{d}{ }^{\min }
$$

Where, $\mathrm{V}_{\mathrm{d}}^{\min }=-0.5 \mathrm{Pg}^{\min }, \mathrm{V}_{\mathrm{d}}{ }^{\max }=+0.5 \mathrm{Pg}_{\mathrm{g}}{ }^{\max }$

\section{SIMULATION AND RESULTS}

\section{Case (a) Thermal Generation}

The ON/OFF schedule obtained by Stage One is given in Table 1.

Table1-ON/OFF Schedule for Case (a)

\begin{tabular}{|l|l|l|l|l|l|l|l|l|l|r|}
\hline Hrs. & \multicolumn{10}{|c|}{ Unit No } \\
\hline & $\mathbf{1}$ & $\mathbf{2}$ & $\mathbf{3}$ & $\mathbf{4}$ & $\mathbf{5}$ & $\mathbf{6}$ & $\mathbf{7}$ & $\mathbf{8}$ & $\mathbf{9}$ & $\mathbf{1 0}$ \\
\hline H1 & 1 & 1 & 0 & 0 & 0 & 0 & 0 & 0 & 0 & 0 \\
\hline H2 & 1 & 1 & 0 & 0 & 0 & 0 & 0 & 0 & 0 & 0 \\
\hline H3 & 1 & 1 & 0 & 0 & 0 & 0 & 0 & 0 & 0 & 0 \\
\hline H4 & 1 & 1 & 0 & 0 & 1 & 0 & 0 & 0 & 0 & 0 \\
\hline H5 & 1 & 1 & 0 & 0 & 1 & 0 & 0 & 0 & 0 & 0 \\
\hline H6 & 1 & 1 & 0 & 1 & 1 & 0 & 0 & 0 & 0 & 0 \\
\hline H7 & 1 & 1 & 1 & 1 & 1 & 0 & 0 & 0 & 0 & 0 \\
\hline H8 & 1 & 1 & 1 & 1 & 1 & 0 & 0 & 0 & 0 & 0 \\
\hline H9 & 1 & 1 & 1 & 1 & 1 & 1 & 0 & 0 & 0 & 0 \\
\hline H10 & 1 & 1 & 1 & 1 & 1 & 1 & 1 & 0 & 0 & 0 \\
\hline H11 & 1 & 1 & 1 & 1 & 1 & 1 & 1 & 1 & 0 & 0 \\
\hline H12 & 1 & 1 & 1 & 1 & 1 & 1 & 1 & 1 & 1 & 0 \\
\hline H13 & 1 & 1 & 1 & 1 & 1 & 1 & 1 & 0 & 0 & 0 \\
\hline H14 & 1 & 1 & 1 & 1 & 1 & 1 & 0 & 0 & 0 & 0 \\
\hline H15 & 1 & 1 & 1 & 1 & 1 & 0 & 0 & 0 & 0 & 0 \\
\hline H16 & 1 & 1 & 1 & 1 & 1 & 0 & 0 & 0 & 0 & 0 \\
\hline H17 & 1 & 1 & 1 & 1 & 1 & 0 & 0 & 0 & 0 & 0 \\
\hline H18 & 1 & 1 & 1 & 1 & 1 & 0 & 0 & 0 & 0 & 0 \\
\hline H19 & 1 & 1 & 1 & 1 & 1 & 0 & 0 & 0 & 0 & 0 \\
\hline H20 & 1 & 1 & 1 & 1 & 1 & 1 & 1 & 0 & 0 & 0 \\
\hline H21 & 1 & 1 & 0 & 1 & 1 & 1 & 1 & 0 & 0 & 0 \\
\hline H22 & 1 & 1 & 0 & 0 & 1 & 1 & 1 & 0 & 0 & 0 \\
\hline H23 & 1 & 1 & 0 & 0 & 0 & 1 & 0 & 0 & 0 & 0 \\
\hline H24 & 1 & 1 & 0 & 0 & 0 & 0 & 0 & 0 & 0 & 0 \\
\hline
\end{tabular}

The economic allocation of load obtained by Stage Two is given in Table 2 . 
Table 2-Economic Load Dispatch Case (a)

\begin{tabular}{|c|c|c|c|c|c|c|c|c|c|c|c|}
\hline \multirow[b]{2}{*}{ Hrs } & \multicolumn{10}{|c|}{ Unit No. } & \multirow{2}{*}{$\begin{array}{l}\text { Tot. } \\
\text { Gen. } \\
\text { (MW) }\end{array}$} \\
\hline & 1 & 2 & 3 & 4 & 5 & 6 & 7 & 8 & 9 & 10 & \\
\hline H1 & 455 & 245 & 0 & 0 & 0 & 0 & 0 & 0 & 0 & 0 & 700 \\
\hline H2 & 455 & 295 & 0 & 0 & 0 & 0 & 0 & 0 & 0 & 0 & 750 \\
\hline H3 & 455 & 395 & 0 & 0 & 0 & 0 & 0 & 0 & 0 & 0 & 850 \\
\hline H4 & 455 & 455 & 0 & 0 & 40 & 0 & 0 & 0 & 0 & 0 & 950 \\
\hline H5 & 455 & 455 & 0 & 0 & 90 & 0 & 0 & 0 & 0 & 0 & 1000 \\
\hline H6 & 455 & 455 & 0 & 130 & 60 & 0 & 0 & 0 & 0 & 0 & 1100 \\
\hline H7 & 455 & 410 & 130 & 130 & 25 & 0 & 0 & 0 & 0 & 0 & 1150 \\
\hline H8 & 455 & 455 & 130 & 130 & 30 & 0 & 0 & 0 & 0 & 0 & 1200 \\
\hline H9 & 455 & 455 & 130 & 130 & 110 & 20 & 0 & 0 & 0 & 0 & 1300 \\
\hline H10 & 455 & 455 & 130 & 130 & 162 & 43 & 25 & 0 & 0 & 0 & 1400 \\
\hline H11 & 455 & 455 & 130 & 130 & 162 & 80 & 25 & 13 & 0 & 0 & 1450 \\
\hline H12 & 455 & 455 & 130 & 130 & 162 & 80 & 25 & 53 & 10 & 0 & 1500 \\
\hline H13 & 455 & 455 & 130 & 130 & 162 & 43 & 25 & 0 & 0 & 0 & 1400 \\
\hline H14 & 455 & 455 & 130 & 130 & 110 & 20 & 0 & 0 & 0 & 0 & 1300 \\
\hline H15 & 455 & 455 & 130 & 130 & 30 & 0 & 0 & 0 & 0 & 0 & 1200 \\
\hline H16 & 455 & 310 & 130 & 130 & 25 & 0 & 0 & 0 & 0 & 0 & 1050 \\
\hline H17 & 455 & 260 & 130 & 130 & 25 & 0 & 0 & 0 & 0 & 0 & 1000 \\
\hline H18 & 455 & 360 & 130 & 130 & 25 & 0 & 0 & 0 & 0 & 0 & 1100 \\
\hline H19 & 455 & 455 & 130 & 130 & 30 & 0 & 0 & 0 & 0 & 0 & 1200 \\
\hline H20 & 455 & 455 & 130 & 130 & 162 & 43 & 25 & 0 & 0 & 0 & 1400 \\
\hline H21 & 455 & 455 & 0 & 130 & 162 & 73 & 25 & 0 & 0 & 0 & 1300 \\
\hline H22 & 455 & 455 & 0 & 0 & 145 & 20 & 25 & 0 & 0 & 0 & 1100 \\
\hline H23 & 455 & 425 & 0 & 0 & 0 & 20 & 0 & 0 & 0 & 0 & 900 \\
\hline H24 & 455 & 345 & 0 & 0 & 0 & 0 & 0 & 0 & 0 & 0 & 800 \\
\hline
\end{tabular}

The generation in MWh and operating costs details (fuel cost and start-up costs)are given in Table3.

Table 3-Operating Cost for Individual Generators for 24 hours for Case (a)

\begin{tabular}{|c|c|c|c|}
\hline Unit No. & Gen. (MWh) & Fuel Cost (\$) & Start-up Cost (\$) \\
\hline $\mathbf{1}$ & 10920 & 203180 & 0 \\
\hline $\mathbf{2}$ & 9870 & 194928.7 & 0 \\
\hline $\mathbf{3}$ & 1820 & 40485.2 & 1100 \\
\hline $\mathbf{4}$ & 2080 & 45770.54 & 1120 \\
\hline $\mathbf{5}$ & 1717 & 43255.23 & 900 \\
\hline $\mathbf{6}$ & 442 & 13718.89 & 510 \\
\hline $\mathbf{7}$ & 175 & 8249.063 & 1040 \\
\hline $\mathbf{8}$ & 66 & 3043.019 & 60 \\
\hline $\mathbf{9}$ & 10 & 937.922 & 60 \\
\hline $\mathbf{1 0}$ & 0 & 0 & 0 \\
\hline Total & $\mathbf{2 7 1 0 0}$ & $\mathbf{5 5 3 5 6 9}$ & $\mathbf{4 7 9 0}$ \\
\hline
\end{tabular}

The convergence of the proposed method is shown in Fig.2. 


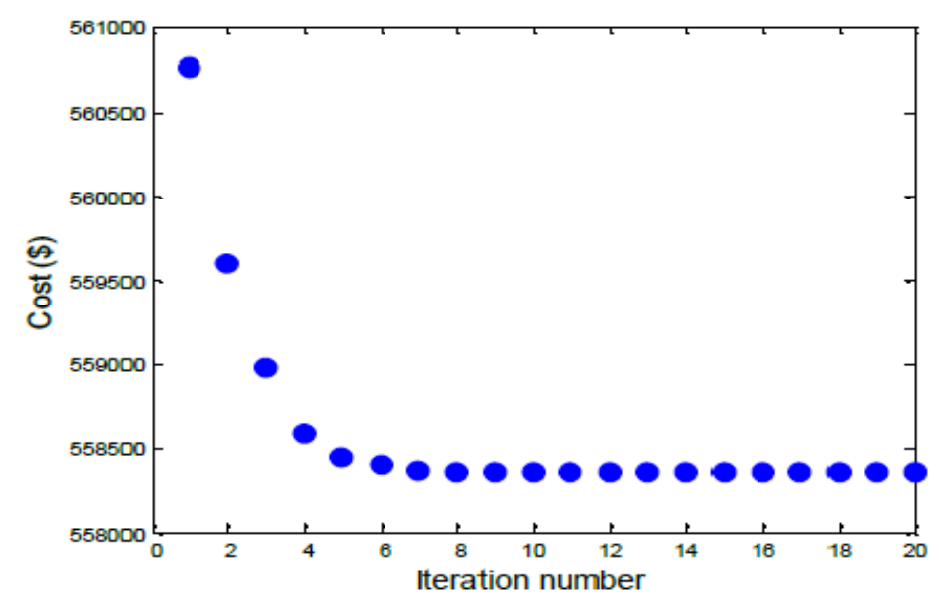

Fig.2. Convergence for Case (a)

The total operating cost for Case (a) is $\mathbf{5 5 8 3 5 9 \$}$.

Case (b) Solar Integrated Thermal Generation

The hourly generation from solar plant is shown in Fig.3.

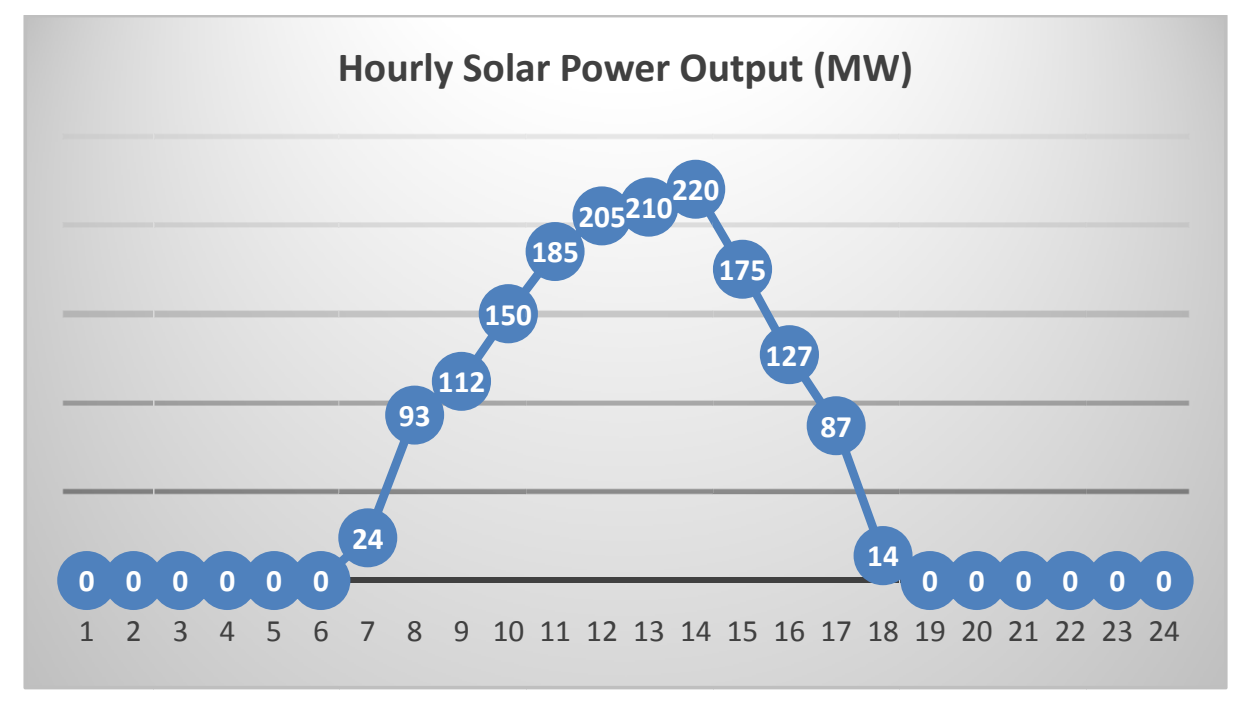

Fig.3. 24 hours Generation from Solar Plant

After calculating the generation from a 300 MW Solar power plant the demand is further updated by subtracting the hourly solar power from the demand. This updated load demand is fed to Stage One to obtain the ON/OFF schedule.

The ON/OFF schedule, Economic allocation of load, operational cost for generation and convergence of the proposed method for Case (b) is given in Table 4, Table 5, Table 6 and Fig.4 respectively. 
Table4-ON/OFF Schedule for Case (b)

\begin{tabular}{|c|c|c|c|c|c|c|c|c|c|c|}
\hline & \multicolumn{10}{|c|}{ Unit No. } \\
\cline { 2 - 12 } Hrs. & $\mathbf{1}$ & $\mathbf{2}$ & $\mathbf{3}$ & $\mathbf{4}$ & $\mathbf{5}$ & $\mathbf{6}$ & $\mathbf{7}$ & $\mathbf{8}$ & $\mathbf{9}$ & $\mathbf{1 0}$ \\
\hline $\mathbf{1}$ & 1 & 1 & 0 & 0 & 0 & 0 & 0 & 0 & 0 & 0 \\
\hline $\mathbf{2}$ & 1 & 1 & 0 & 0 & 0 & 0 & 0 & 0 & 0 & 0 \\
\hline $\mathbf{3}$ & 1 & 1 & 0 & 0 & 0 & 0 & 0 & 0 & 0 & 0 \\
\hline $\mathbf{4}$ & 1 & 1 & 0 & 0 & 1 & 0 & 0 & 0 & 0 & 0 \\
\hline $\mathbf{5}$ & 1 & 1 & 0 & 1 & 1 & 0 & 0 & 0 & 0 & 0 \\
\hline $\mathbf{6}$ & 1 & 1 & 0 & 1 & 1 & 0 & 0 & 0 & 0 & 0 \\
\hline $\mathbf{7}$ & 1 & 1 & 0 & 1 & 1 & 0 & 0 & 0 & 0 & 0 \\
\hline $\mathbf{8}$ & 1 & 1 & 0 & 1 & 1 & 0 & 0 & 0 & 0 & 0 \\
\hline $\mathbf{9}$ & 1 & 1 & 1 & 1 & 1 & 0 & 0 & 0 & 0 & 0 \\
\hline $\mathbf{1 0}$ & 1 & 1 & 1 & 1 & 1 & 0 & 0 & 0 & 0 & 0 \\
\hline $\mathbf{1 1}$ & 1 & 1 & 1 & 1 & 1 & 0 & 0 & 0 & 0 & 0 \\
\hline $\mathbf{1 2}$ & 1 & 1 & 1 & 1 & 1 & 1 & 0 & 0 & 0 & 0 \\
\hline $\mathbf{1 3}$ & 1 & 1 & 1 & 0 & 1 & 1 & 0 & 0 & 0 & 0 \\
\hline $\mathbf{1 4}$ & 1 & 1 & 0 & 0 & 1 & 1 & 0 & 0 & 0 & 0 \\
\hline $\mathbf{1 5}$ & 1 & 1 & 0 & 0 & 1 & 1 & 0 & 0 & 0 & 0 \\
\hline $\mathbf{1 6}$ & 1 & 1 & 0 & 0 & 1 & 0 & 0 & 0 & 0 & 0 \\
\hline $\mathbf{1 7}$ & 1 & 1 & 0 & 0 & 1 & 0 & 0 & 0 & 0 & 0 \\
\hline $\mathbf{1 8}$ & 1 & 1 & 0 & 1 & 1 & 0 & 0 & 0 & 0 & 0 \\
\hline $\mathbf{1 9}$ & 1 & 1 & 1 & 1 & 1 & 1 & 0 & 0 & 0 & 0 \\
\hline $\mathbf{2 0}$ & 1 & 1 & 1 & 1 & 1 & 1 & 0 & 1 & 1 & 0 \\
\hline $\mathbf{2 1}$ & 1 & 1 & 1 & 1 & 1 & 1 & 0 & 0 & 0 & 0 \\
\hline $\mathbf{2 2}$ & 1 & 1 & 1 & 1 & 0 & 0 & 0 & 0 & 0 & 0 \\
\hline $\mathbf{2 3}$ & 1 & 1 & 1 & 0 & 0 & 0 & 0 & 0 & 0 & 0 \\
\hline $\mathbf{2 4}$ & 1 & 1 & 0 & 0 & 0 & 0 & 0 & 0 & 0 & 0 \\
\hline
\end{tabular}

Table 5-Economic Load Dispatch Case (b)

\begin{tabular}{|c|c|c|c|c|c|c|c|c|c|c|c|c|}
\hline \multirow{2}{*}{ Hrs. } & \multicolumn{10}{|c|}{ Unit No. } & $\begin{array}{c}\text { Tot. } \\
\text { Gen. } \\
\text { (MW) }\end{array}$ \\
\hline $\mathbf{1}$ & $\mathbf{1}$ & $\mathbf{2}$ & $\mathbf{3}$ & $\mathbf{4}$ & $\mathbf{5}$ & $\mathbf{6}$ & $\mathbf{7}$ & $\mathbf{8}$ & $\mathbf{9}$ & $\mathbf{1 0}$ & $\mathbf{P s}_{\text {olar }}$ & \\
\hline $\mathbf{2}$ & 455 & 245 & 0 & 0 & 0 & 0 & 0 & 0 & 0 & 0 & 0 & $\mathbf{7 0 0}$ \\
\hline $\mathbf{3}$ & 455 & 395 & 0 & 0 & 0 & 0 & 0 & 0 & 0 & 0 & 0 & $\mathbf{7 5 0}$ \\
\hline $\mathbf{4}$ & 455 & 455 & 0 & 0 & 40 & 0 & 0 & 0 & 0 & 0 & 0 & $\mathbf{9 5 0}$ \\
\hline $\mathbf{5}$ & 455 & 390 & 0 & 130 & 25 & 0 & 0 & 0 & 0 & 0 & 0 & $\mathbf{1 0 0 0}$ \\
\hline $\mathbf{6}$ & 455 & 455 & 0 & 130 & 60 & 0 & 0 & 0 & 0 & 0 & 0 & $\mathbf{1 1 0 0}$ \\
\hline $\mathbf{7}$ & 455 & 455 & 0 & 130 & 86 & 0 & 0 & 0 & 0 & 0 & 24 & $\mathbf{1 1 5 0}$ \\
\hline $\mathbf{8}$ & 455 & 455 & 0 & 130 & 67 & 0 & 0 & 0 & 0 & 0 & 93 & $\mathbf{1 2 0 0}$ \\
\hline $\mathbf{9}$ & 455 & 448 & 130 & 130 & 25 & 0 & 0 & 0 & 0 & 0 & 112 & $\mathbf{1 3 0 0}$ \\
\hline $\mathbf{1 0}$ & 455 & 455 & 130 & 130 & 80 & 0 & 0 & 0 & 0 & 0 & 150 & $\mathbf{1 4 0 0}$ \\
\hline $\mathbf{1 1}$ & 455 & 455 & 130 & 130 & 95 & 0 & 0 & 0 & 0 & 0 & 185 & $\mathbf{1 4 5 0}$ \\
\hline $\mathbf{1 2}$ & 455 & 455 & 130 & 130 & 105 & 20 & 0 & 0 & 0 & 0 & 205 & $\mathbf{1 5 0 0}$ \\
\hline $\mathbf{1 3}$ & 455 & 455 & 130 & 0 & 130 & 20 & 0 & 0 & 0 & 0 & 210 & $\mathbf{1 4 0 0}$ \\
\hline $\mathbf{1 4}$ & 455 & 455 & 0 & 0 & 150 & 20 & 0 & 0 & 0 & 0 & 220 & $\mathbf{1 3 0 0}$ \\
\hline $\mathbf{1 5}$ & 455 & 455 & 0 & 0 & 95 & 20 & 0 & 0 & 0 & 0 & 175 & $\mathbf{1 2 0 0}$ \\
\hline $\mathbf{1 6}$ & 455 & 443 & 0 & 0 & 25 & 0 & 0 & 0 & 0 & 0 & 127 & $\mathbf{1 0 5 0}$ \\
\hline $\mathbf{1 7}$ & 455 & 433 & 0 & 0 & 25 & 0 & 0 & 0 & 0 & 0 & 87 & $\mathbf{1 0 0 0}$ \\
\hline $\mathbf{1 8}$ & 455 & 455 & 0 & 130 & 46 & 0 & 0 & 0 & 0 & 0 & 14 & $\mathbf{1 1 0 0}$ \\
\hline
\end{tabular}




\begin{tabular}{|c|c|c|c|c|c|c|c|c|c|c|c|c|}
\hline $\mathbf{1 9}$ & 455 & 440 & 130 & 130 & 25 & 20 & 0 & 0 & 0 & 0 & 0 & $\mathbf{1 2 0 0}$ \\
\hline $\mathbf{2 0}$ & 455 & 455 & 130 & 130 & 162 & 48 & 0 & 10 & 10 & 0 & 0 & $\mathbf{1 4 0 0}$ \\
\hline $\mathbf{2 1}$ & 455 & 455 & 130 & 130 & 110 & 20 & 0 & 0 & 0 & 0 & 0 & $\mathbf{1 3 0 0}$ \\
\hline $\mathbf{2 2}$ & 455 & 385 & 130 & 130 & 0 & 0 & 0 & 0 & 0 & 0 & 0 & $\mathbf{1 1 0 0}$ \\
\hline $\mathbf{2 3}$ & 455 & 315 & 130 & 0 & 0 & 0 & 0 & 0 & 0 & 0 & 0 & $\mathbf{9 0 0}$ \\
\hline $\mathbf{2 4}$ & 455 & 345 & 0 & 0 & 0 & 0 & 0 & 0 & 0 & 0 & 0 & $\mathbf{8 0 0}$ \\
\hline
\end{tabular}

Table 6-Operating Cost for Individual Generators for 24 hours for Case (b)

\begin{tabular}{|c|c|c|c|}
\hline Unit No. & Gen. (MWh) & Fuel Cost (\$) & Start-up Cost (\$) \\
\hline $\mathbf{1}$ & 10920 & 203180 & 0 \\
\hline $\mathbf{2}$ & 10049 & 198056 & 0 \\
\hline $\mathbf{3}$ & 1300 & 28918 & 1650 \\
\hline $\mathbf{4}$ & 1690 & 37188.6 & 1120 \\
\hline $\mathbf{5}$ & 1351 & 35254.2 & 900 \\
\hline $\mathbf{6}$ & 168 & 6363.17 & 510 \\
\hline $\mathbf{7}$ & 0 & 0 & 0 \\
\hline $\mathbf{8}$ & 10 & 919.613 & 60 \\
\hline $\mathbf{9}$ & 10 & 937.922 & 60 \\
\hline $\mathbf{1 0}$ & 0 & 0 & 0 \\
\hline Total & $\mathbf{2 5 4 9 8}$ & $\mathbf{5 1 0 8 1 8}$ & $\mathbf{4 3 0 0}$ \\
\hline
\end{tabular}

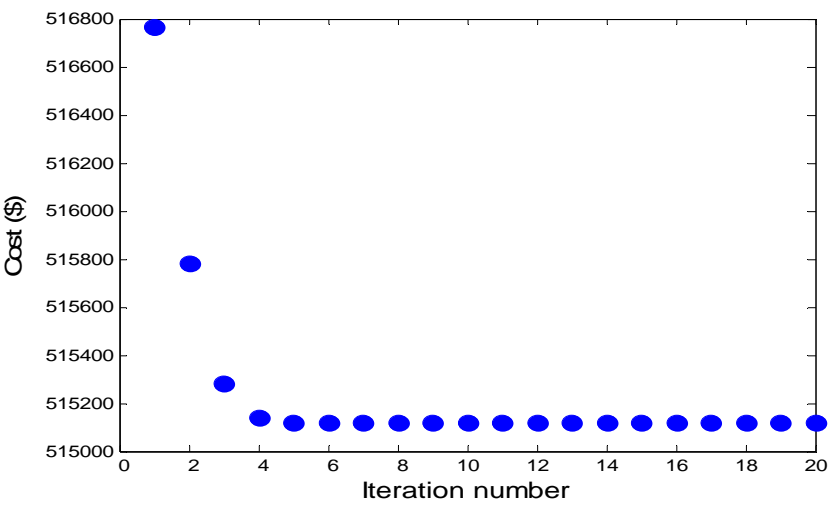

Fig.4. Convergence for Case (b)

The total operating cost for Case (a) is $\mathbf{5 1 5 1 1 8}$ \$.

\section{CONCLUSION}

It can be concluded from this work that after properly scheduling renewable energy resources with conventional thermal generation the fuel cost of thermal generation can be significantly reduced. In this case after inclusion of a 300 MW Solar generation plant the total saving in fuel cost is $\mathbf{4 3 2 4 1 \$}$ per day. The hybridization of Priority List Method (PLM) with Particle Swarm Optimization Technique with Time Varying Acceleration Coefficients (PSO_TVAC) gives satisfactory results for a non-linear, complex and constraints based problem like UCP. The convergence of the proposed method remains unaltered even after inclusion of renewable generation. 
APPENDICES

Appendix I (Thermal Units Detail)[5, 14]

\begin{tabular}{|c|c|c|c|c|c|c|c|c|c|}
\hline Unit No. & 1 & 2 & 3 & 4 & 6 & 7 & 8 & 9 & 10 \\
\hline$P_{\max }$ & 455 & 455 & 130 & 130 & 80 & 85 & 55 & 55 & 55 \\
\hline $\mathrm{P}_{\min }$ & 150 & 150 & 20 & 20 & 20 & 25 & 10 & 10 & 10 \\
\hline$a(\$ / h)$ & 1000 & 970 & 700 & 680 & 370 & 480 & 660 & 665 & 670 \\
\hline $\mathrm{b}(\$ / \mathrm{MWh})$ & 16.19 & 17.26 & 16.60 & 16.50 & 22.26 & 27.74 & 25.92 & 27.27 & 27.79 \\
\hline$c\left(\$ / M^{2} h\right)$ & 0.00048 & 0.00031 & 0.002 & 0.00211 & 0.0072 & 0.00079 & 0.00413 & 0.0022 & 0.00173 \\
\hline $\mathrm{MD}(\mathrm{h})$ & 8 & 8 & 5 & 5 & 3 & 3 & 1 & 1 & 1 \\
\hline $\mathrm{MU}(\mathrm{h})$ & 8 & 8 & 5 & 5 & 3 & 3 & 1 & 1 & 1 \\
\hline $\mathrm{HSc}(\$ / \mathrm{h})$ & 4500 & 5000 & 550 & 560 & 170 & 260 & 30 & 30 & 30 \\
\hline $\operatorname{CSc}(\$ / h)$ & 9000 & 10000 & 1100 & 1120 & 340 & 520 & 60 & 60 & 60 \\
\hline $\mathrm{Cs}(\mathrm{h})$ & 5 & 5 & 4 & 4 & 2 & 2 & 0 & 0 & 0 \\
\hline $\begin{array}{l}\text { Initial } \\
\text { Status }\end{array}$ & 8 & 8 & -5 & -5 & -3 & -3 & -1 & -1 & -1 \\
\hline
\end{tabular}

Appendix II (Load Profile for 24 Hours)[5, 14]

\begin{tabular}{|c|c|c|c|c|c|c|}
\hline Hour & H1 & $\mathrm{H} 2$ & H3 & H4 & H5 & H6 \\
\hline $\begin{array}{l}\text { Load } \\
\text { Mrw) }\end{array}$ & 700 & 750 & 850 & 950 & 1000 & 1100 \\
\hline Hour & H7 & Hs & $\mathrm{Ho}$ & $\mathrm{H} 10$ & H 11 & $\mathrm{H} 12$ \\
\hline $\begin{array}{l}\text { Load } \\
\text { (Mrw) }\end{array}$ & 1150 & 1200 & 1300 & 1400 & 1450 & 1500 \\
\hline Hour & $\mathrm{H} 13$ & $\mathrm{H} 14$ & H15 & H16 & H17 & H1s \\
\hline $\begin{array}{l}\text { Load } \\
\text { (Mry) }\end{array}$ & 1400 & 1300 & 1200 & 1050 & 1000 & 1100 \\
\hline Hour & H19 & $\mathrm{H} 2 \mathrm{O}$ & $\mathrm{H} 21$ & $\mathrm{H} 22$ & H23 & $\mathrm{H} 24$ \\
\hline $\begin{array}{l}\text { Load } \\
\text { (Mrw) }\end{array}$ & 1200 & 1400 & 1300 & 1100 & 900 & 800 \\
\hline
\end{tabular}




\section{Appendix III (Solar Radiation Data for 24 Hours)[5]}

\begin{tabular}{|c|c|c|c|c|c|c|}
\hline Hour & 1 & 2 & 3 & 4 & 5 & 6 \\
\hline $\mathrm{SR}\left(\mathrm{N} / \mathrm{m}^{2}\right)$ & 0 & 0 & 0 & 0 & 0 & 0 \\
\hline Hour & 7 & 8 & 9 & 10 & 11 & 12 \\
\hline $\operatorname{SR}\left(\mathbb{N} / \mathbf{m}^{2}\right)$ & 111 & 311 & 375 & 503 & 617 & 686 \\
\hline Hour & 13 & 14 & 15 & 16 & 17 & 18 \\
\hline $\operatorname{SR}\left(\mathbb{W} / \mathbf{m}^{\mathbf{2}}\right)$ & 703 & 736 & 586 & 425 & 291 & 86 \\
\hline Houlr & 19 & 20 & 21 & 22 & 23 & 24 \\
\hline $\operatorname{SR}\left(\mathbb{N} / \mathbf{m}^{2}\right)$ & 0 & 0 & 0 & 0 & 0 & 0 \\
\hline
\end{tabular}

\section{REFERENCES}

[1] Wood A. J. and Wollenberg B. F, “Power generation, operation and control”, John Wiley \& sons, New York, $3^{\text {rd }}$ Edition.

[2] Vine E, "Breaking down the silos: the integration of energy efficiency, renewable energy, demand response and climate changes", Energy efficiency, 1:49-63, 2008.

[3] Juan M. Morales, Antonio J. Conejo, Henrik Madsen, Pierre Pinson and Marco Zugno, "Integrating Renewables in Electricity Markets: operational problems”, International series in Operational Research\& Management Science, Springer, 2014.

[4] Anup Shukla and S.N Singh, "PSO for solving Unit Commitment Problem including Renewable Energy Resources”, Electrical India, Vol. 54, No. 12, pp. 100-105, Dec. 2014.

[5] Shantanu C.,Senjyu T.,Saber A.Y.,Yona A. and Funabashi T., "Optimal Thermal Unit Commitment Integrated with Renewable Energy Sources Using Advanced Particle Swarm Optimization”, IEEJ Trans.4,pp.609-617, 2009.

[6] Anup Shukla, S.N Singh, “Multi-objective Unit Commitment with Renewable Energy using GSA Algorithm”, Springer, INAE Letters,vol.1,issue 1,pp.21-27,2016.

[7] Anup Shukla, S. N. Singh, "Multi-objective Unit Commitment Using Search Space Based Crazy PSO and Normal Boundary Intersection Technique", IET, Generation,Transmission and Distribution, Vol. 10, Issue 5, pp. 1222-1231, Apr. 2016.

[8] Shubham Tiwari, Ankit Kumar, G.S. Chaurasia, G.S. Sirohi, “Economic Load Dispatch Using Particle Swarm Optimization”, IJAIEM, Vol. 2, Issue 4, pp 476-485, 2013.

[9] Pappala VS and Erlich I., “A new approach for solving Unit Commitment Problem by adaptive particle swarm optimization”, Power and Energy Society general meeting-conversion and delivery of electrical energy in the $21^{\text {st }}$ century, USA:IEEE ,pp.1-6., 2008.

[10] Sayeed Salem, “Unit Commitment Solution Methods”,World Academy of Science, Engineering and Technology, pp.320-325, 2007

[11] Shubham Tiwari, Bharti Dwivedi and M.P Dave, "Advancements in Unit Commitment Strategy", In: proceedings of IEEE International Conference on Emerging Trends in Electrical, Electronics \& Sustainable Energy Systems (ICETEESES-16) ,Vol.1,Issue 1, pp.259-262,11-12 March,2016.

[12] Yun-Won Jeong, Jong-Bae Park, Se-Hwan Jang, and Kwang Y. Lee, “A New Quantum-Inspired Binary PSO: Application to Unit Commitment Problems for Power Systems”, IEEE Transactions On Power Systems, Vol. 25, No. 3, Aug. 2010 ,pp.1486-95.

[13] Anup Shukla, S.N. Singh, “Advanced three stage pseudo inspired weight improved crazy particle swarm optimization for Unit Commitment Problem”,Elseveir, Energy, Vol.96, pp.23-36, Feb. 2016.

[14] Shubham Tiwari, Bharti Dwivedi, M.P Dave, “A Two stage solution methodology for Deterministic Unit Commitment problem”, In:Proceedings of $3^{\text {rd }}$ IEEE International Conference on Electrical, Computer and Electronics( UPCON-2016),09-11 Dec., 2016,p.p.317-322.

[15] Archana Yadav, Shubham Tiwari and Bharti Dwivedi, “A hybrid PSO based technique for Unit Commitment problem”, In:Proceedings of IEEE International Conference on Computing, Communication and Control Technology (IC ${ }^{4}$ T-2016), Vol.1,Issue 1,pp.191-195,Nov. 2016.

[16] Khanmohammadi S., Amiri M. and Tarafdar Haque M., “A new three stage method for solving unit commitment method”, Elsevier Energy 35(2010),3072-3080

[17] K.T Chaturvedi, Manjaree Pandit, Laxmi Srivastava,“Self-Organizing Hierarchical Particle Swarm Optimization for Non-Convex Economic Dispatch”; IEEE Transactions on Power Systems,August2008,vol.23,pp.1079-1087.

[18] Shubham Tiwari, Abhishek Maurya, "Particle Swarm Optimization Technique with Time Varying Acceleration coefficients for load Dispatch Problem”, IJRITCC, Vol. 3, Issue 6, Jun. 2015, pp. 3878-3885. 